PALABRAS CLAVE

Educación

Ingresos

Distribución del ingreso

Salarios

Igualdad

Mercado de trabajo

Etnicidad

Clases sociales

Perú

\section{¿Mejora la distribución del ingreso con la educación? El caso del Perú}

\author{
Adolfo Figueroa
}

i

el sistema educativo un factor nivelador del ingreso? Según datos provenientes de países en desarrollo, si bien la educación se ha expandido enormemente en los últimos decenios, la distribución del ingreso no se ha vuelto más equitativa. En el presente artículo se procura resolver esta aparente paradoja. Se construye un modelo teórico sobre la relación entre educación e ingresos en el que la identidad étnica desempeña un papel clave en el proceso de distribución. El modelo permite diagnosticar que el sistema educativo no es un factor nivelador de los ingresos. A partir del modelo teórico se deriva un conjunto más amplio de predicciones, que posteriormente se contrastan con información disponible respecto del Perú. Los resultados de las pruebas estadísticas coinciden con las predicciones del modelo. 


\section{I}

\section{Introducción}

Durante los últimos decenios la educación ha experimentado una enorme expansión en los países en desarrollo. De acuerdo con datos del Banco Mundial, en el período comprendido entre 1980 y 1997 la tasa neta de matrícula de la enseñanza primaria se elevó del $78 \%$ al $89 \%$, mientras que en la enseñanza secundaria dicha tasa subió del 53\% al 63\% (Banco Mundial, 2001, cuadro 6, pág. 285). Sin embargo, paradójicamente no ha disminuido el grado de desigualdad del ingreso (Deininger y Squire, 1996, cuadro 5; Li, Squire y Zou, 1998). Cabe entonces preguntarse por qué la educación no es un sistema nivelador del ingreso.

La bibliografía disponible no ha proporcionado una explicación científica satisfactoria para esta paradoja y los modelos teóricos más difundidos sobre movilidad intergeneracional (Becker y Tomes, 1979; Durlauf, 1996) no han sido estadísticamente corroborados en países en desarrollo.

En cambio, hay numerosos trabajos empíricos sobre las relaciones entre educación y desigualdad del ingreso en el mundo en desarrollo, pero estos carecen de sustentación teórica. En el caso especial de América Latina, a diferencia de la situación de los países más avanzados es posible observar tres regularidades empíricas: i) medida en función de los años de escolaridad, la distribución de la educación es más desigual; ii) la brecha de calidad entre las escuelas a que asisten alumnos pobres y ricos es mayor, y iii) la diferencia de ingresos entre los trabajadores de alto y bajo nivel de educación es mucho más amplia, lo que algunos autores atribuyen a la oferta relativamente limitada de trabajadores instruidos (Bourguignon,
Ferreira y Menéndez, 2007; Birdsall, De la Torre y Menezes, 2008; Blom y Vélez, 2004).

En este artículo se examina el papel que desempeñan la clase social y la identidad étnica de los individuos para explicar las relaciones entre educación e ingreso, incluida la paradoja ya señalada. Para ello se desarrolla un modelo teórico sencillo, cuyas predicciones se contrastan con los datos sobre el Perú. En la bibliografía internacional no existen muchas referencias al papel de la identidad étnica en las relaciones entre la educación y los ingresos en países en desarrollo. En el caso del Perú, Nopo, Saavedra y Torero (2004) encontraron que ella cumple una función importante en la descomposición estadística de las diferencias de los salarios urbanos. Con respecto al Brasil, en el estudio de Bourguignon, Ferreira y Menéndez (2007) antes citado también se encontró que la raza influye en las diferencias de ingresos. Sin embargo, ninguno de los dos estudios tiene sustento teórico.

El artículo se estructura de la siguiente forma: en la sección II se presenta un modelo teórico en que la clase y la identidad étnica juegan un papel importante en el proceso educativo. La sección III se refiere al papel de la desigualdad inicial en la dotación de activos de las familias en el proceso de acumulación de capital humano. En las secciones IV y V se presentan modelos estáticos y dinámicos. En la sección VI se comprueban estadísticamente las predicciones empíricas del modelo con los datos para el Perú, utilizando pruebas paramétricas y no paramétricas (que se describen detalladamente en el Apéndice). En la sección VII se entregan las conclusiones. 


\section{II}

\section{Un modelo teórico sencillo}

Considérese una sociedad capitalista hipotética en que la distribución de los activos económicos y sociales de las personas es altamente desigual. Se parte de la base de que las personas participan en el proceso económico dotadas no solo de activos económicos, sino también sociales, con lo que se introducen factores sociales en el proceso económico. Los activos sociales son especiales porque pertenecen al ámbito de los derechos y titularidades que se otorgan a las personas en una sociedad. Se trata de bienes que no son materiales ni comerciables.

En el presente trabajo la referencia a activos sociales se relaciona básicamente con bienes políticos y culturales. Los primeros se definen como la capacidad de las personas de ejercer derechos individuales y colectivos, incluido el de tener derechos. El hecho de que las dotaciones individuales de activos políticos sean desiguales genera en la sociedad una jerarquía de ciudadanos: ciudadanos de primera y de segunda clase. A consecuencia de ello, no todas las personas son iguales ante la ley; es más, no todas las personas tienen igual acceso a los bienes públicos proporcionados por el Estado. ${ }^{1}$

Los bienes culturales se definen como el derecho de los grupos sociales a ejercer la diversidad cultural en una sociedad multicultural y multiétnica. La dotación desigual de derechos culturales da lugar a que existan grupos étnicos que tienen una jerarquía de indicadores étnicos en la sociedad: razas, idiomas, religiones y costumbres de primera y de segunda clase. Se dice que estos indicadores son culturales porque su jerarquía se establece socialmente y se transmite de generación en generación. La desigualdad en materia de activos culturales conduce a prácticas sociales de segregación, exclusión y discriminación de determinados grupos étnicos.

En el trabajo se parte del supuesto de que la desigualdad en las dotaciones individuales de activos políticos y culturales está positivamente correlacionada en la sociedad, de modo que en la teoría se puede incluir solamente la desigualdad en los activos políticos. Para

\footnotetext{
${ }^{1}$ El concepto de ciudadanía utilizado en este trabajo corresponde al propuesto por el sociólogo Marshall en su clásico ensayo de 1950, en que sostiene que la ciudadanía es una condición otorgada a quienes son miembros plenos de una comunidad y que todos los que poseen esta condición son iguales respecto de los derechos y deberes de que ella está provista (Marshall y Bottomore, 1992, pág. 18).
}

mayor facilidad, se puede llamar "sociedad sigma" a esta sociedad ficticia.

Una de las características de la sociedad sigma es la desigual distribución de los activos económicos y políticos. La otra característica se relaciona con la dotación de factores: la existencia de sobrepoblación. Este supuesto implica que la productividad marginal del total de la fuerza de trabajo es demasiado baja como para que las tasas de salarios permitan equilibrar la demanda y la oferta en los mercados de trabajo.

A fin de derivar predicciones empíricamente rebatibles de la teoría sigma hay que construir una sociedad sigma determinada o un modelo específico de la teoría sigma. A continuación se introduce un conjunto de supuestos auxiliares.

La estructura social de la sociedad sigma se compone de raza, clase y ciudadanía. La población total puede dividirse en: i) dos clases sociales: capitalistas y trabajadores; ii) dos tipos de ciudadanos: de primera y de segunda clase, y iii) tres grupos étnicos: los azules, los rojos y los morados, estos últimos resultantes del cruce de las otras dos razas. ${ }^{2}$

En el cuadro 1 se muestra la estructura social de la sociedad sigma en forma de matriz. Los azules son la clase capitalista, dueña de la mayor parte del capital físico. Los morados y los rojos pertenecen a la clase trabajadora. Los morados tienen mano de obra calificada y los rojos no calificada. En cuanto a los derechos de ciudadanía, los azules y los morados son ciudadanos de primera clase, mientras que los rojos son de segunda clase, con lo que se establece la desigualdad inicial en materia de dotación inicial de activos. En la matriz social se observa una sociedad altamente correlacionada en lo que atañe a dotación de activos. En consecuencia, pueden identificarse tres grupos. Para facilidad de referencia y por razones que se evidenciarán más adelante, los grupos se denominarán con las letras A, Y y Z.

\footnotetext{
${ }^{2}$ En un trabajo en que se examinan las relaciones teóricas entre las preferencias de los consumidores y la cultura, Akerlof y Kranton (2000) construyen un mundo abstracto en que hay dos grupos étnicos, los verdes y los rojos, donde los verdes son el grupo dominante. Para utilizar colores primarios, aquí se llamarán azules y rojos y luego se introducirá un tercer grupo étnico, los morados, resultante del entrecruzamiento de las dos razas. $\mathrm{Al}$ igual que en ese trabajo, aquí se parte de la base de que las personas no pueden elegir su identidad étnica, que es exógena.
} 
CUADRO 1

Estructura social de la sociedad signa: raza, clase, ciudadanía

\begin{tabular}{lcccc}
\hline $\begin{array}{l}\text { Grupo } \\
\text { éntico }\end{array}$ & $\begin{array}{c}\text { Capital } \\
\text { físico }\end{array}$ & $\begin{array}{c}\text { Capital } \\
\text { humano }\end{array}$ & Ciudadanía & $\begin{array}{r}\text { Grupo } \\
\text { social }\end{array}$ \\
\hline Azules & $\mathrm{K}_{\mathrm{b}}$ & $\mathrm{K}_{\mathrm{h} 1}$ & $\mathrm{C}_{1}$ & $\mathrm{~A}$ \\
Morados & 0 & $\mathrm{~K}_{\mathrm{h} 1}$ & $\mathrm{C}_{1}$ & $\mathrm{Y}$ \\
Rojos & 0 & $\mathrm{~K}_{\mathrm{h} 0}$ & $\mathrm{C}_{0}$ & $\mathrm{Z}$ \\
\hline
\end{tabular}

Fuente: elaboración propia.

Símbolos: $\mathrm{K}_{\mathrm{b}}$ dotación de capital físico; $\mathrm{K}_{\mathrm{h} 1}$ dotación de capital humano de alto nivel; $\mathrm{K}_{\mathrm{h} 0}$ dotación de capital humano de bajo nivel; $\mathrm{C}_{1}$ dotación de ciudadanía de primera clase; $\mathrm{C}_{0}$ dotación de ciudadanía de segunda clase.

Para fines analíticos, la sociedad sigma puede ahora distinguirse de una sociedad capitalista socialmente homogénea, que podría denominarse "sociedad épsilon".
Si esta se representara por el cuadro 1 , de todos modos habría dos clases sociales (capitalistas y trabajadores), pero una sola clase de ciudadanía (C). Siempre habría tres grupos étnicos, pero un solo nivel de ciudadanía para todos (C). En consecuencia, en la sociedad épsilon las diferencias raciales carecerían de importancia y la matriz social se reduciría a tan solo dos grupos sociales: A (capitalistas) e Y (trabajadores), y no existiría el grupo social Z. Mientras que se supone que la sociedad sigma se asemeja al mundo en desarrollo, la sociedad épsilon corresponde al mundo desarrollado.

La cuestión que se examina en este trabajo es si el proceso de acumulación de capital humano mediante la educación puede reducir la desigualdad inicial en la distribución de activos (incluido naturalmente el capital humano) y determinar de esta manera que los flujos de ingresos puedan hacerse menos desiguales. La respuesta a esta interrogante se desarrolla en el resto del trabajo.

\section{III}

\section{La acumulación de capital humano: el papel que desempeña la dotación inicial de activos}

El capital humano se define generalmente como el acervo de conocimientos y destrezas productivas que están incorporados en los trabajadores. Puesto que las personas no adquieren este capital al nacer, deben invertir para adquirirlo mediante el proceso educativo.

En el proceso educativo, las condiciones iniciales que trae el invidividuo al sistema educativo (en cada nivel educacional) son esenciales para el aprendizaje. En la bibliografía de las ciencias conexas (psicología, biología y neurociencias) frecuentemente se postula que la dotación inicial de talentos es importante y que ellos son múltiples: la denominada "teoría de la inteligencia múltiple" (Gardner, 1999).

Otro componente importante del enfoque tradicional es la teoría de la plasticidad del cerebro. Mientras que los talentos de que dispone inicialmente la persona —su patrimonio genético- son exógenos (naturales), con el tiempo ellos se tornan endógenos porque el desarrollo del cerebro también depende del medio social (crianza) y de la acción recíproca entre ambos. La teoría de la plasticidad del cerebro se formula habitualmente de la siguiente manera: el cerebro no es un computador que se limita a ejecutar programas predeterminados. Tampoco es una col madura, víctima de las influencias ambientales que la afectan. Los genes y el medio interactúan permanentemente para modificar el cerebro, desde la concepción hasta la muerte (Ratey, 2002, pág. 17).

En general, puede partirse de la base de que la dotación individual de talentos, dones de base genética, se distribuye normalmente entre la población (resultado de un mecanismo aleatorio). En cambio, la distribución de las destrezas de aprendizaje será generada por el medio social. La importante distinción hecha por Rousseau (1755) se refiere precisamente a estos dos factores. Este autor identificó dos tipos de desigualdades entre las personas: la desigualdad natural, determinada por factores naturales asignados al azar, y la desigualdad artificial, determinada por el funcionamiento de la sociedad.

De acuerdo con el modelo sigma, se presumirá que la nutrición, la salud y el lenguaje son los principales canales a través de los cuales las familias adineradas pueden desarrollar en sus hijos niveles más altos de destrezas de aprendizaje en comparación con los de las familias más pobres. Se supone que el acceso personal 
a la nutrición y la salud, sea como bienes privados o públicos, se diferencia según el grupo social a que pertenece cada individuo.

El lenguaje es otro factor de desigualdad en materia de destrezas cognitivas vinculado al nivel socioeconómico de la familia. En una sociedad sigma, estas diferencias se traducen en una desigualdad en el manejo del lenguaje. Esta desigualdad se comprueba en diversos aspectos del uso del lenguaje en el idioma dominante en la sociedad, como son el vocabulario, la sintaxis, las maneras de hablar y las destrezas de lectura y escritura. De acuerdo con la teoría sociolingüística, la desigualdad de lenguaje obedece principalmente a experiencias personales (el medio social) y no a factores genéticos (Hudson, 1996, pág. 204).

La desigualdad en materia de destrezas en el uso del lenguaje entre los grupos sociales implica desigualdad de destrezas cognitivas entre los hijos de estos. El pensamiento abstracto y complejo necesita no solo del lenguaje, sino de un lenguaje complejo. Como lo señaló el filósofo John Searle, algunos pensamientos son de tal complejidad que sería empíricamente imposible pensarlos sin poseer símbolos. Por ejemplo, el pensamiento matemático requiere un sistema de símbolos. El pensamiento abstracto complejo exige palabras y símbolos (Searle, 1995, pág. 64). De ello parece desprenderse que el lenguaje escrito permite que la persona trabaje con pensamientos más abstractos y complejos que si utiliza únicamente el lenguaje oral.

Considérese una situación en que los trabajadores $Y$ viven en una cultura escrita y la mayoría de ellos sabe leer y escribir, mientras que los trabajadores $Z$ viven en un medio social oral, son en su mayoría analfabetos en el idioma dominante y su lenguaje aborigen no es escrito. Supóngase además que los trabajadores $\mathrm{Z}$ viven en una cultura oral en comunidades segregadas. En un escenario de este tipo, las destrezas de manejo del lenguaje en el idioma dominante se distribuirán muy desigualmente a través de la sociedad.
Debido a su analfabetismo, en las poblaciones $\mathrm{Z}$ el uso del pensamiento abstracto y complejo será limitado. Ser analfabeto en una cultura escrita es distinto a serlo en una cultura oral. La desventaja del analfabeto será mayor en la primera. En consecuencia, las destrezas lingüísticas en el idioma dominante de las poblaciones $\mathrm{Z}$ serán menores y por lo tanto sus hijos tendrán niveles más bajos de destrezas cognitivas que los hijos de las poblaciones A e Y.

El hecho de ser una sociedad multicultural, multilingüe y jerárquica hace que la sociedad sigma sea heteroglósica. Este término proviene de la teoría sociolingüística y se refiere a la existencia de diversas formas de uso del idioma dominante o variaciones de este que establecen una jerarquía que separa a las personas socialmente superiores (que manejan bien el lenguaje dominante) de aquellas consideradas inferiores.

A continuación, en el modelo sigma se presupone que la desigualdad de lenguaje desempeña un papel fundamental en la generación de destrezas cognitivas desiguales. La teoría sociolingüística va incluso más lejos al sostener que la desigualdad lingüística podría considerarse una de las causas de la desigualdad social, a la vez que una consecuencia de ella, porque el lenguaje es uno de los medios más importantes por cuyo intermedio se perpetúa la desigualdad social de generación en generación (Hudson, 1996, pág. 205).

En consecuencia, en una sociedad sigma —en la que al comienzo hay una pronunciada desigualdad en cuanto a la dotación de activos económicos y políticos, y dada la distribución aleatoria de talentos en la población- las personas iniciarán el proceso de acumulación de capital humano provistas de distintas destrezas cognitivas o de aprendizaje. Esta desigualdad inicial cumple una función importante en el proceso de acumulación de capital humano entre los grupos sociales. Cabe señalar que esta proposición no es empíricamente refutable o falseable debido a que la capacidad de aprendizaje no puede observarse y, por lo tanto, se utilizará como un supuesto primario de la teoría de acumulación de capital humano que se presenta a continuación. 


\section{IV}

\section{La transformación de la educación en ingreso: un modelo estático}

El modelo estático sigma puede representarse mediante el siguiente sistema de ecuaciones:

$$
\begin{aligned}
& k_{h}=f(E, X), f_{1}>0, f_{2}>0, X=Z, Y, A \\
& y=g\left(k_{h}, X, p\right), g_{1}>0, g_{2}>0, g_{3}>0
\end{aligned}
$$

Luego

$$
\begin{aligned}
& y=g(f(E, X), X, p) \\
& =G(E, X, p), G_{1}>0, G_{2}>0, G_{3}>0
\end{aligned}
$$

De acuerdo con la ecuación (1), en promedio la dotación de capital humano de las personas $\left(k_{h}\right)$ depende del nivel medio de educación medido en años de escolaridad $(E)$ y del grupo social a que pertenecen $(Z, Y, A)$. En un grupo social determinado, un aumento exógeno del nivel medio de educación se traducirá en un nivel medio más alto de capital humano. En un nivel de educación determinado, las personas que pertenecen a grupos sociales de mayor rango (en los que la jerarquía en orden ascendente es Z, Y, A) tendrán un nivel medio más alto de capital humano.

En la ecuación (2) se establece que el ingreso medio de las personas $(y)$ depende de su disponibilidad media de capital humano y del grupo social a que pertenecen en determinadas condiciones de mercado $(p)$. En un grupo social determinado, mientras mayor sea la media de capital humano, mayor será el ingreso medio. En determinadas condiciones de disponibilidad de capital humano, el ingreso medio será mayor en el caso de las personas que pertenecen a grupos sociales de mayor rango (en orden ascendente: $\mathrm{Z}, \mathrm{Y}, \mathrm{A}$ ).

Para simplificar, supóngase que las condiciones del mercado dependen de los términos de intercambio a nivel internacional $(p)$, que a corto plazo son la única variable exógena que determina el nivel agregado de producto, empleo e ingreso medio. Un valor más alto de $p$ significa que el valor de los bienes exportados es superior al de las importaciones, lo que a su vez indica que la productividad de la mano de obra es mayor y que también lo es la demanda de mano de obra de las empresas nacionales. Por consiguiente, en determinados niveles de educación, mientras más alto sea el valor de $p$, mayor será el ingreso medio de los grupos sociales.

De acuerdo con la teoría sigma, se supone que la estructura social emana de la desigualdad inicial de la distribución de los activos económicos y políticos. Esta desigualdad — la variable $\delta$ (delta) — subyace al término $X$ de la ecuación (3). Por lo tanto, las diferencias de ingreso entre grupos sociales dependen de las diferencias en el nivel medio de educación y la dotación de activos. Mientras los valores de las variables exógenas $E$ y $\delta$ permanezcan constantes, el ingreso medio de cada grupo social continuará invariable y lo mismo sucederá con la distribución del ingreso a través de los grupos sociales.

En el gráfico 1 se muestra el modelo estático. Los puntos $m, n$ y $r$ son los valores medios de los años de escolaridad de los tres grupos sociales A, Y y Z, y los puntos $m^{\prime}, n^{\prime}$ y $r^{\prime}$ son los valores correspondientes del ingreso medio. La desigualdad inicial de la distribución de activos da lugar a una situación en que la clase capitalista tiene más años de escolaridad, capital humano e ingresos, seguida de los otros dos grupos sociales. Además, los años adicionales de escolaridad del grupo Z se traducirán en un nivel de ingresos mayor a lo largo de la curva Z. El mismo proceso se aplica a los grupos $\mathrm{Y}$ y A. La curva L, observable empíricamente, representa la situación de equilibrio.

En la situación de equilibrio representada en el gráfico 1, para analizar el efecto de las variaciones exógenas en educación en la desigualdad del ingreso pueden considerarse tres casos. Primero, un año más de escolaridad en el grupo Z por sí solo elevará el ingreso medio del grupo y aminorará la desigualdad inicial, pero el efecto será reducido. Segundo, un año más de escolaridad en todos los grupos sociales tendrá escasos efectos en la desigualdad: ciertamente aumentará el ingreso medio en todos los grupos, pero no hay indicaciones de que los ingresos relativos vayan a cambiar de manera significativa. En tercer lugar, el efecto de los años adicionales de escolaridad en los tres grupos, con mayor acento en el grupo Z, será ambiguo y, en el mejor de los casos, pequeño. 
GRÁFICO 1

Hipótesis de la relación entre educación (E), ingreso medio (y) y grupos étnicos

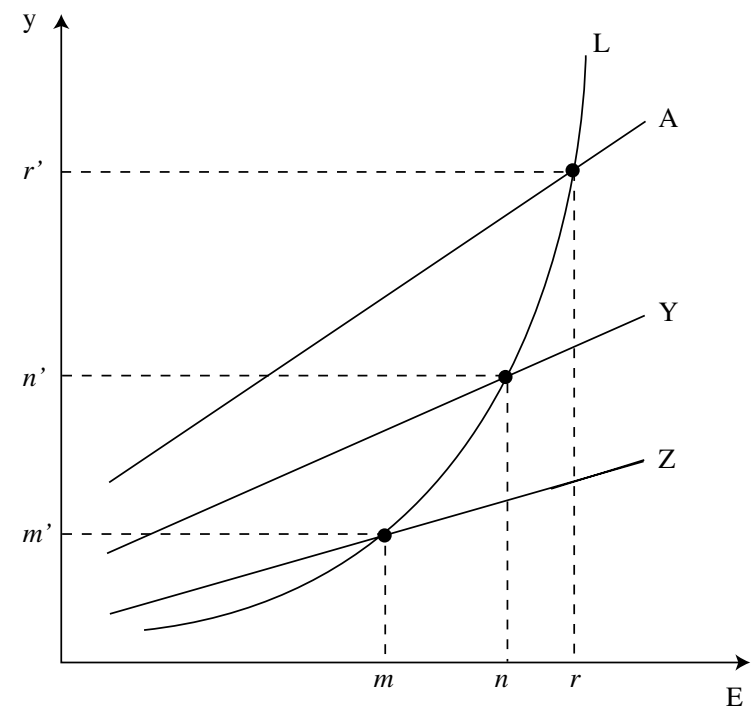

Fuente: elaboración propia.

En síntesis, el modelo estático permite predecir que la reducción de la desigualdad con los años de escolaridad no implica una baja significativa de la desigualdad. Es más, el mismo número de años de escolaridad no genera un promedio de ingresos igual para todos los grupos sociales. Ello obedece a que la diferencia en materia de años de escolaridad es solo uno de los factores que generan diferencias de ingreso entre los grupos sociales.

La conclusión sería diferente si las relaciones entre los años de escolaridad y el ingreso no fueran separables, esto es, si solo existiese una curva para todos los grupos. Si las tres curvas se redujeran a la curva A del gráfico 1, un año adicional de escolaridad aumentaría el ingreso en igual magnitud en los tres grupos sociales. Por lo tanto, si se redujeran las diferencias en años de escolaridad también disminuiría la desigualdad de los ingresos. Además, un mismo número de años de escolaridad generaría el mismo promedio de ingresos para todos los grupos sociales. Sin embargo, de acuerdo con el modelo sigma, esta no es la forma en que opera el sistema capitalista. La sociedad sigma tiene tres curvas separadas (relaciones de clase y raza), mientras que la sociedad épsilon solo tendría dos curvas separadas (relaciones de clase).

Las relaciones que figuran en el gráfico 1 se refieren a los valores medios de las variables correspondientes a cada grupo social. Es posible que se den casos de éxito (personas que se trasladan de la curva $\mathrm{Z}$ a Y, o incluso A), pero de acuerdo con la teoría esos casos serán más bien la excepción y no la regla.

Como lo indica el signo de sus efectos en la ecuación (3), los cambios en las variables exógenas modificarán de determinadas maneras los ingresos medios de los grupos sociales. Así se representan las predicciones empíricas del modelo en la ecuación (3), que son empíricamente refutables y pueden utilizarse para poner a prueba según mediciones estadísticas la validez del modelo. La curva $L$ del gráfico 1 relaciona los valores medios de los años de escolaridad y los ingresos de cada grupo social. En consecuencia, la situación de equilibrio estático que aparece en el gráfico 1 se representa por la curva L.

El modelo sigma se aparta de diversas maneras del análisis estándar. Por lo general, en los estudios empíricos convencionales se miden las relaciones entre educación e ingresos a lo largo de la curva L. Sin embargo, en el modelo sigma no se puede utilizar la curva L para comparar los efectos de los cambios exógenos en los años de escolaridad en los ingresos, ni las tasas de rentabilidad de la educación (como erradamente lo hacen algunos estudios internacionales), porque los efectos de la educación en los ingresos operan en trayectorias diferentes (A, Y, Z) en los distintos grupos sociales. Si la relación se midiera sobre la curva L, ciertamente se sobrestimaría el efecto de la educación en los ingresos.

También hay que señalar que este modelo se relaciona con el análisis por grupos cuyo objeto es explicar las diferencias de ingresos y de educación a través de los grupos sociales. En cambio, en la bibliografía convencional se examina la relación empírica entre ingresos y educación aplicando el modelo de regresiones de Mincer (Mincer, 1974), que se refiere al análisis individual y apunta por lo tanto a estudiar las diferencias en materia de ingresos y educación entre las personas (incluidas en las regresiones variables como experiencia, edad, género y otras características individuales). 


\section{V}

\section{Un modelo dinámico}

Supóngase ahora que a largo plazo la cantidad de educación se determina en forma endógena en la sociedad y en cada grupo social. Supóngase, además, que se conocen las condiciones iniciales del proceso económico, en otras palabras, que las dotaciones individuales de activos económicos y políticos son desiguales desde la partida y que ellas determinan la estructura social A, Y y Z. Supóngase, también, que en este modelo de largo plazo la relación de intercambio internacional se mantiene invariable.

El equilibrio dinámico se definirá ahora como una secuencia de situaciones de equilibrio estático en el tiempo. Por consiguiente, se considerará ahora que la situación de equilibrio estático es la solución del período o generación inicial, lo que es dado por la curva L del gráfico 1. En esta curva se muestran los valores iniciales de educación e ingreso por cada grupo social, lo que implica valores medios de educación e ingresos para la sociedad en su conjunto.

En el modelo dinámico se supone que una parte del producto total obtenido en este período o generación se asignará a inversión en capital humano en la forma de años de escolaridad. Esta inversión es financiada por las familias y por el Estado mediante políticas fiscales, según el grupo social de que se trate. Como consecuencia de ello, en el segundo período habrá más educación y capital humano, ya que aumentará el ingreso medio de la sociedad. Para simplificar, en el modelo se parte de la base de que el crecimiento del ingreso medio depende fundamentalmente del incremento del capital humano y, por lo tanto, también de la expansión de la educación. Se supone que los demás factores que generalmente se considera que influyen en el crecimiento económico - como la acumulación de capital físico y el cambio tecnológico- son inducidos por los niveles más altos de educación.

Considérese ahora el proceso dinámico por grupos sociales. En el siguiente período o generación, los niveles de educación y el ingreso medio serán mayores en cada grupo social (y en el agregado de la sociedad); habrá nuevas inversiones, que en el período subsiguiente se traducirán en niveles aun mayores de educación e ingreso medio en cada grupo social (y en el agregado), y así sucesivamente. Este proceso dinámico puede modelarse mediante la curva L del gráfico 1 . Dado el equilibrio inicial, la inversión en educación implicará un desplazamiento de la curva hacia el exterior, por ejemplo a L1, lo que por su parte significará un equilibrio nuevo en que cada grupo social obtendrá mayores ingresos. En este período se invertirá más en educación y la curva volverá a desplazarse hacia el exterior, por ejemplo a L2, y así sucesivamente. En suma, con el tiempo la curva $L$ se desplazará endógenamente hacia el exterior.

En el proceso de acumulación de capital humano mediante la educación, cada grupo social se trasladará a lo largo de su trayectoria específica dada por las líneas Z, Y y A del gráfico 1. Según se dijo, el proceso de expansión de la educación a lo largo de sus correspondientes trayectorias no genera reducciones significativas de la desigualdad de ingresos entre los grupos sociales. Las diferencias en materia de años de escolaridad pueden aminorarse, no así las disparidades de ingresos. Dada la desigualdad inicial en materia de dotación de activos, hay dos razones por las que los ingresos no convergen: i) al transformar la educación en capital humano, una mayor igualdad en años de escolaridad no significa que se logre mayor igualdad en capital humano, puesto que los grupos sociales acumulan ese capital siguiendo trayectorias diferentes; ii) al convertir el capital humano en ingresos, el ingreso depende del acceso a los mercados básicos, que varía según el grupo social de que se trate, y esta relación no varía mediante la acumulación de capital humano. Esto quiere decir que la educación se transforma en ingresos siguiendo trayectorias diferentes, según el grupo social de que se trate. Los ingresos relativos de los tres grupos sociales dependen de las condiciones iniciales de la sociedad, de su historia. En otras palabras, las condiciones iniciales de la sociedad son importantes, la historia social cuenta.

En consecuencia, la desigualdad inicial en materia de dotación de activos económicos y políticos es clave para explicar por qué el sistema educativo no es nivelador. Con el tiempo, es posible que se eliminen las diferencias en años de escolaridad, porque estos tienen un tope, pero la desigualdad de ingresos no disminuye de manera importante, ya que a lo largo del tiempo los grupos sociales siguen trayectorias diferentes. La movilidad educacional es posible, pero la movilidad socioeconómica es mucho más problemática. 
Expresado en términos intergeneracionales, en el modelo dinámico sigma se predice que los "hijos" de un grupo social determinado tienden a heredar la condición económica de sus "padres". Hace mucho tiempo, el biólogo inglés Francis Dalton formuló la "ley de regresión hacia la media" (tendencia hacia la igualdad) respecto de las diferencias de estatura entre los hijos y sus padres biológicos. En el modelo sigma que se ofrece en este trabajo se predice que en los ingresos entre "hijos" y "padres" del mismo grupo social no habrá "regresión hacia la media".

El modelo dinámico sigma puede explicar la paradoja de la siguiente manera: las sociedades de los países en desarrollo son socialmente heterogéneas y jerárquicas, es decir, se parecen a la sociedad sigma. La desigualdad en la distribución inicial de los activos económicos y políticos hace que la sociedad funcione con exclusiones. En especial, los procesos para transformar la educación en capital humano y este en ingresos se diferencian según relaciones de clase y etnia. En los países desarrollados la desigualdad inicial es tal que estos procesos se diferencian solamente por las relaciones de clase, pero en el mundo en desarrollo la identidad étnica también desempeña un papel importante en la reproducción de la desigualdad. Mientras la desigualdad inicial en materia de dotación de activos permanezca invariable, el sistema educativo no será nivelador de los ingresos.

\section{VI}

\section{Hipótesis empíricas confrontadas con los datos sobre el Perú}

Del modelo estático sigma puede derivarse un conjunto de predicciones empíricas sobre las relaciones entre educación e ingresos. A continuación se confrontarán estas predicciones con los datos disponibles sobre el Perú, sociedad multiétnica y multicultural, para ver si ellas contradicen o corroboran al modelo. Los datos corresponden a la Encuesta Nacional de Hogares (ENAHO) realizada en 2003.

Estimar en forma empírica el tamaño de los grupos étnicos es una tarea difícil. En el caso del Perú, la población $\mathrm{Z}$ corresponde a los descendientes de pueblos indígenas. Para medir el tamaño de estos grupos se consideraron hasta cuatro criterios, todos los cuales tienen inconvenientes, a saber:

- Criterio relacionado con la lengua materna: en este criterio se reduce la "etnicidad" al indicador de lenguaje, por lo que subestima su tamaño. Así, no podrían considerarse indígenas los pueblos originarios que no hablan una lengua aborigen, sea porque pertenecen a un grupo que no ha heredado una lengua aborigen o a una generación que ha perdido dicha lengua.

- Criterio de la autoidentificación: en este enfoque se subestima el tamaño de la población indígena, porque en una sociedad jerárquica las personas tienden a ocultar su identidad étnica.

- Criterio de la residencia en un medio rural: también se subestima el tamaño de la población indígena, porque no podrían considerarse indígenas las personas que emigran a la ciudad.

- Criterio del nacimiento en un distrito rural: en este caso se subestima una vez más el tamaño de la población indígena, porque no se considerarían indígenas los hijos nacidos en una ciudad (después de la migración).

En el presente trabajo se eligió el último de estos criterios para estimar el tamaño de la población indígena, puesto que parece ser el más confiable. Además, el análisis solo se referirá a la población adulta, de 25 años o más, de modo que las personas nacidas en distritos rurales del Perú de las tres regiones naturales - la costa, la región andina y la región amazónica-, sea donde fuere que residan ahora, se considerarán mayormente indígenas. Este constituye el grupo social Z del modelo teórico.

Se estima que las personas nacidas en los distritos residenciales más adinerados de Lima, sea cual fuere el lugar en que residen ahora, pertenecen mayormente al grupo social A. El grupo mestizo o Y se calculó como la diferencia entre los otros dos. Las estimaciones son las siguientes: el grupo social A representa un 3,5\% del total de la población adulta; el grupo Y, un $27,5 \%$, y el grupo $\mathrm{Z}$, un $69 \%$. $^{3}$

\footnotetext{
${ }^{3}$ En la Encuesta Nacional de Hogares (ENAHO) de 2001 se incluía una pregunta sobre la autoidentificación étnica. El resultado fue que,
} 


\section{H1. Hipótesis de las diferencias cuantitativas en materia de años de escolaridad entre los grupos sociales}

Como se observa en el gráfico 1, el modelo estático sigma predice que el promedio de años de escolaridad varía según el grupo social y aumenta en el orden Z, Y, A. Esta relación se muestra en el cuadro 2. La media de años de escolaridad varía entre grupos sociales: 14 años para el grupo A, 11 años en el caso del grupo Y y 7 para el grupo Z; las medianas se dan en el mismo orden: 14 años, 11 años y 5 años. En las pruebas estadísticas paramétricas y no paramétricas (véase el Apéndice) se aprecia que las diferencias observadas en los valores medios son estadísticamente significativas. Los datos empíricos no refutan la hipótesis; ellos ponen de manifiesto una pronunciada desigualdad en materia de educación.

Al utilizar los niveles de educación (básica, secundaria, técnica y universitaria) en vez de los años de escolaridad, también queda de manifiesto una marcada desigualdad. Casi el 70\% del grupo A tiene educación post secundaria, comparado con el $36 \%$ del grupo Y y solo un $15 \%$ del grupo Z: el promedio nacional es del $21 \%$. La exclusión del grupo $\mathrm{Z}$ de la educación post secundaria es un hecho notable.

Son escasos los estudios sobre la conversión de la educación en capital humano por grupo social. Los que existen presentan resultados comparados de pruebas de rendimiento escolar realizadas a un grado determinado, entre dos categorías de escuelas: público-privadas y urbano-rurales. Los resultados revelan que el promedio de conocimientos, medido por los resultados de las pruebas, es superior entre los alumnos de escuelas privadas que entre los de las escuelas públicas y también en las escuelas urbanas respecto de las rurales (Rivera, 1979; Cueto, Jacoby y Pollit, 1997; Perú, Ministerio de Educación, 2005).

También hay algunas indicaciones del papel negativo que desempeña la desnutrición en el proceso de aprendizaje escolar. En un investigación realizada en el Programa de Estudios Conjuntos de Integración Económica Latinoamericana (ECIEL), basada en la

entre los jefes de hogar, un $43 \%$ se declaró descendiente de indígenas, mientras que un 34\% señaló que hablaba una lengua aborigen (Hall y Patrinos, 2006, cuadros 2.1, 2.7 y 7.1). Como era de esperar a partir de las definiciones utilizadas, estas estimaciones de la proporción de personas indígenas son claramente inferiores al $69 \%$ calculado en este estudio. Dado el orden de magnitud de estas estimaciones, lo afirmado por numerosos autores peruanos en el sentido de que el grupo étnico predominante es Y ("somos un país de mestizos") carece de apoyo empírico. población escolar de Lima y la de Puno (separando entre urbano y rural), resalta que hay una correlación negativa amplia y estadísticamente significativa entre los rendimientos escolares de los niños y la desnutrición (Rivera, 1979).

Como en las zonas rurales predomina el grupo social $Z$, hay que deducir que en el Perú la transformación de la educación en capital humano parece funcionar como lo indica la ecuación estructural (1). Estos resultados son consistentes con la hipótesis. Tras 180 años como república, y pese a la expansión del sistema educativo, la población indígena del Perú aún se encuentra significativamente al margen del sistema educativo, tanto cuantitativa como cualitativamente.

\section{H2. Hipótesis de la "separabilidad" y jerarquía de la relación entre educación e ingresos}

En el modelo estático sigma se indica que existe una relación positiva entre la media de años de escolaridad y el ingreso medio, pero que esta relación es separable y jerárquica por grupos sociales en orden ascendente Z, Y, A. Como se muestra en la ecuación (3) y en el gráfico 1, esto es precisamente lo que sostiene la forma reducida del modelo. En consecuencia, para corroborar el modelo sigma epistemológicamente se justifica utilizar la ecuación en su forma reducida y no es necesario hacerlo mediante las ecuaciones estructurales. En otras palabras, si la ecuación (3) fuera falsa, los supuestos contenidos en las ecuaciones (1) y (2) no podrían ser verdaderos.

En el cuadro 3 se entregan datos sobre los ingresos por nivel de educación y grupo social. En este caso, la población se refiere a la población económicamente activa (PEA) que está empleada (no se tiene en cuenta al grupo reducido de desempleados). Los datos observados sugieren que el ingreso medio y el nivel de educación están directamente vinculados, tanto en el interior de los grupos sociales como entre ellos. Lo que hay que resolver es si las relaciones observadas pueden separarse estadísticamente para cada grupo social y si en ellas se da una jerarquía A, Y, Z, como lo propone la hipótesis.

Dada la existencia de exclusión educativa cuantitativa, que confirma la hipótesis $H 1$, algunos de los casilleros del cuadro 3 contienen cifras muy pequeñas. Por consiguiente, la prueba solo debe realizarse entre los niveles de educación comparables.. Los miembros del grupo $\mathrm{Z}$ se concentran en los primeros niveles de educación y solo una pequeña proporción, inferior al $10 \%$, alcanza niveles post secundarios. En el grupo $\mathrm{Y}$ hay una proporción reducida de analfabetos. En 
CUADRO 2

Perú: nivel de educación por grupo social, 2003

(En miles de personas y porcentajes)

\begin{tabular}{|c|c|c|c|c|c|c|c|c|}
\hline \multirow{3}{*}{ Nivel de educación } & \multicolumn{6}{|c|}{ Grupo social } & \multirow{2}{*}{\multicolumn{2}{|c|}{ Total }} \\
\hline & \multicolumn{2}{|c|}{$\mathrm{Z}$} & \multicolumn{2}{|c|}{$\mathrm{Y}$} & \multicolumn{2}{|c|}{ A } & & \\
\hline & $\mathrm{N}^{\mathrm{a}}$ & (porcentaje) & $\mathrm{N}$ & (porcentaje) & $\mathrm{N}$ & (porcentaje) & $\mathrm{N}$ & (porcentaje) \\
\hline Ninguno & 1283 & 14,1 & $110^{\mathrm{b}}$ & 3,3 & $0,7^{\mathrm{b}}$ & 0,2 & 1394 & 10,9 \\
\hline Básica & 3903 & 43 & 556 & 16,5 & $10,1^{\mathrm{b}}$ & 2,8 & 4470 & 34,9 \\
\hline Técnica & $725^{b}$ & 8 & 530 & 15,7 & 73,4 & 20,8 & 1328 & 10,4 \\
\hline Universitaria & $562^{b}$ & 6,2 & 697 & 20,7 & 174,4 & 49,3 & 1433 & 11,2 \\
\hline Total N & 9083 & 100 & 3368 & 100 & 353,4 & 100 & 12805 & 100 \\
\hline Porcentaje horizontal & 70,9 & & 26,3 & & 2,8 & & 100 & \\
\hline \multicolumn{9}{|l|}{ Años de escolaridad } \\
\hline Promedio & 6,9 & & 10,8 & & 13,7 & & 8,2 & \\
\hline
\end{tabular}

Fuente: elaboración propia sobre la base de Encuesta Nacional de Hogares 2003.

a Población de 25 años o más (ampliación de la muestra).

b Número reducido de observaciones, un $10 \%$ o menos del grupo social.

CUADRO 3

Perú: ingreso medio por nivel de educación y grupo social, 2003

(En miles de personas, soles por mes y porcentajes)

\begin{tabular}{|c|c|c|c|c|c|c|c|c|c|}
\hline \multirow{3}{*}{ Nivel de educación } & \multicolumn{6}{|c|}{ Grupo social } & \multirow{2}{*}{\multicolumn{3}{|c|}{ Total }} \\
\hline & \multicolumn{2}{|l|}{$\mathrm{Z}$} & \multicolumn{2}{|l|}{$\mathrm{Y}$} & \multicolumn{2}{|l|}{ A } & & & \\
\hline & $\begin{array}{c}\mathrm{N}^{\mathrm{a}} \\
\text { (porcentaje) }\end{array}$ & $\mathrm{y}^{\mathrm{b}}$ & $\begin{array}{c}\mathrm{N} \\
\text { (porcentaje) }\end{array}$ & $\mathrm{y}$ & $\stackrel{\mathrm{N}}{\text { (porcentaje) }}$ & $\mathrm{y}$ & $\mathrm{N}$ & (porcentaje) & $\mathrm{y}$ \\
\hline Ninguno & $9,6^{\mathrm{c}}$ & 171 & $1,9^{\mathrm{c}}$ & 297 & - & - & 604 & 7,1 & 180 \\
\hline Básica & 41 & 330 & 13,1 & 479 & $1,0^{\mathrm{c}}$ & 137 & 2704 & 32 & 346 \\
\hline Secundaria & 32,7 & 592 & 44 & 710 & 22,7 & 798 & 2998 & 35,4 & 637 \\
\hline Técnica & $9,6^{\mathrm{c}}$ & 844 & 17,2 & 912 & 15,8 & 965 & 1001 & 11,9 & 876 \\
\hline Universitaria & $7,1^{\mathrm{c}}$ & 1529 & 23,8 & 2005 & 60,5 & 2721 & 1148 & 13,6 & 1943 \\
\hline Total & 100 & 535 & 100 & 1015 & 100 & 1981 & 8464 & 100 & 717 \\
\hline Total N & 5841 & & 2330 & & 293 & & 8464 & & \\
\hline Porcentaje horizontal & 69 & & 27,5 & & 3,5 & & 100 & & \\
\hline \multicolumn{10}{|l|}{ Años de escolaridad } \\
\hline Promedio & 7,6 & & 11,4 & & 14,2 & & 9 & & \\
\hline Mediana & 8 & & 11 & & 15 & & 11 & & \\
\hline
\end{tabular}

Fuente: elaboración propia sobre la base de Encuesta Nacional de Hogares 2003.

a Población de 25 años o más (ampliación de la muestra).

b Ingreso medio en soles, Lima, octubre de 2003.

c Número reducido de observaciones, un $10 \%$ o menos del grupo social.

- No hay población en esta categoría. 
el grupo A no había analfabetos, o las personas solo tenían educación básica. Por lo tanto, los niveles de educación y los grupos sociales comparables o pertinentes son los siguientes: en el nivel básico, los grupos Y y Z; en el nivel secundario, los tres grupos $\mathrm{A}, \mathrm{Y}, \mathrm{Z}, \mathrm{y}$ en los niveles técnico y universitario, los grupos A e Y. En los resultados de las pruebas estadísticas que figuran en el Apéndice se aprecia que las diferencias observadas son generalmente significativas. En resumen, los datos empíricos no refutan la hipótesis $H 2$.

En el gráfico 2 se ilustra la naturaleza de la relación empírica entre los ingresos y la educación y se muestran los niveles correspondientes para fines de comparación. La gradiente de las líneas mostradas indica una tendencia ascendente en los tres grupos sociales. Además, la pendiente de la curva $\mathrm{A}$ se eleva más rápidamente que la de la curva $\mathrm{Y}$, y la de esta más rápido que la de la curva $\mathrm{Z}$.

GRÁFICO 2

\section{Perú: relación empírica entre ingreso medio $(y)$ y nivel de educación, según grupos étnicos}

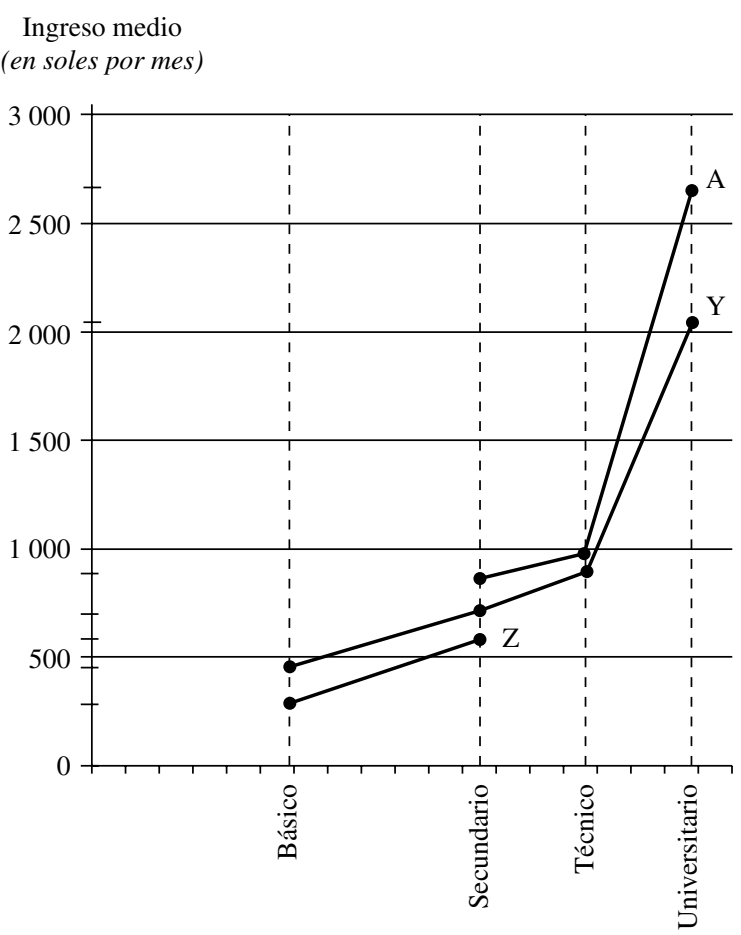

Nivel de educación

Fuente: elaboración propia sobre la base de los datos consignados en el cuadro 2.
Para comprobar econométricamente las relaciones entre las pendientes podría aplicarse a estos datos el método estándar de regresión, utilizando como variables ficticias a los grupos sociales A, Y, Z. Sin embargo, los datos revelan que el único nivel aplicable para fines de comparación es la educación secundaria. Como se sabe, uno de los supuestos implícitos del modelo estándar de regresión consiste en que las variables ficticias deben abarcar toda la gama de observaciones de las variables independientes: si la distribución de estas variables contiene exclusiones, como en el caso del gráfico 2, el modelo estándar de regresión no resulta aplicable. En la práctica, la teoría econométrica estándar parte implícitamente de la base de que en las relaciones entre variables no hay exclusiones.

\section{H3. Hipótesis de la "separabilidad" y jerarquía en las relaciones entre educación y salarios}

Si en el eje vertical se sustituye el ingreso por los salarios, el gráfico 1 también puede representar las relaciones entre la educación y los salarios. En este caso se introducirá una serie de supuestos. Primero, la productividad de la mano de obra depende del nivel de capital humano de los trabajadores y no de su nivel de educación. Segundo, las empresas orientadas a la maximización de las utilidades que operan en mercados laborales competitivos contratarán trabajadores de acuerdo con su productividad y pagarán salarios uniformes para un nivel determinado de capital humano.

La situación de equilibrio estático del mercado laboral puede expresarse de la siguiente manera:

$$
\begin{gathered}
k_{h}=f(E, X), f_{1}>0, f_{2}>0 \\
w=h\left(K_{h}, X, p\right), h_{1}>0, h_{2}>0, h_{3}>0
\end{gathered}
$$

Luego,

$$
\begin{gathered}
w=h(f(E, X), X, p)=H(E, X, p), \\
H_{1}>0, H_{2}>0, H_{3}>0
\end{gathered}
$$

La ecuación (4) corresponde a la anterior ecuación (1) en que se mostraba la transformación de la educación en capital humano. La ecuación (5) también es conocida y ahora se muestra en ella la transformación del capital humano en salarios (w). La ecuación (6) corresponde a la forma reducida que ahora permite ver que en definitiva los salarios dependen de la educación y del grupo social, para un valor de $p$ dado. 
En los cuadros 4A y 4B se ofrecen datos sobre la educación y las remuneraciones en los mercados laborales de obreros y empleados, respectivamente. El sueldo medio de los empleados duplica los salarios de los obreros. La diferencia en materia de educación apunta en la misma dirección: la media de años de escolaridad es de 13,7 años para los empleados y de 8,5 años para los obreros, con medianas de 14 años y 10 años, respectivamente. Los niveles de educación aparecen como factor diferenciador entre los dos grupos sociales. La proporción de trabajadores $\mathrm{Z}$ que se ocupan en el mercado laboral obrero es del $20 \%$, pero solo del $14 \%$ en el de empleados. Dentro del grupo Y, las proporciones equivalentes son del $21 \%$ y el $36 \%$; y en el grupo A, del $8 \%$ y el $62 \%$.

En los niveles de educación pertinentes para comparar entre los obreros (véase el cuadro 4A) se incluyen la enseñanza básica y secundaria y los trabajadores de los grupos Y y Z. En cada caso, el salario medio observado de los trabajadores $\mathrm{Y}$ es superior al de los trabajadores Z. Las pruebas estadísticas indican que estas diferencias son estadísticamente significativas solo en el nivel de educación secundaria (que constituye el grupo más amplio en este mercado de trabajo), como se muestra en el Apéndice. En los niveles de educación pertinentes para comparar entre los empleados (véase el cuadro 4B) se incluyen la enseñanza secundaria, técnica y universitaria y los grupos sociales Z, Y, A. En la comprobación estadística se observa que las diferencias son estadísticamente significativas en cinco de los nueve casos, y en el nivel universitario es sostenidamente significativa, como figura en el Apéndice.

En general, las pruebas estadísticas aquí presentadas revelan que los sueldos y salarios se relacionan directamente con el nivel de educación de los trabajadores. Como los empleados suelen ser más instruidos que los obreros, el sueldo medio de los primeros es superior al salario medio de los segundos. Por último, en un nivel de educación determinado y pertinente, las remuneraciones dependen generalmente de la jerarquía de los grupos sociales, dada por el orden A, Y, Z. La misma relación se observa en el caso de los sueldos de los empleados. En resumen, los datos empíricos ofrecidos no refutan la hipótesis $H 3$.

(En miles de personas, soles por mes y porcentajes)

\begin{tabular}{|c|c|c|c|c|c|c|c|c|}
\hline \multirow{3}{*}{ Nivel de educación } & \multicolumn{6}{|c|}{ Grupo social } & \multirow{2}{*}{\multicolumn{2}{|c|}{ Total }} \\
\hline & \multicolumn{2}{|c|}{$\mathrm{Z}$} & \multicolumn{2}{|c|}{$\mathrm{Y}$} & \multicolumn{2}{|c|}{$\mathrm{A}$} & & \\
\hline & $\mathrm{N}^{\mathrm{a}}(\%)$ & $\mathrm{IM}^{\mathrm{b}}$ & $\mathrm{N}(\%)$ & $\mathrm{IM}^{\mathrm{b}}$ & $\mathrm{N}(\%)$ & $\mathrm{IM}^{\mathrm{b}}$ & $\mathrm{N}(\%)$ & $\mathrm{IM}^{\mathrm{b}}$ \\
\hline Ninguno & $6^{\mathrm{c}}$ & 306 & $1,8^{\mathrm{c}}$ & 349 & - & - & 4,7 & 310 \\
\hline Básica & 37,5 & 489 & 21,6 & 518 & - & - & 32,4 & 490 \\
\hline Secundaria & 46,7 & 616 & 59,4 & 651 & 78,2 & 569 & 50,8 & 627 \\
\hline Técnica & $8,1^{\mathrm{c}}$ & 695 & 13,8 & 712 & 21,8 & 743 & 10 & 703 \\
\hline Universitaria & $1,7^{\mathrm{c}}$ & 763 & $3,4^{\mathrm{c}}$ & 796 & - & - & 2,1 & 778 \\
\hline Total & 100 & & 100 & & 100 & 606 & 100 & 579 \\
\hline Total N & 1163 & & 482 & & 23 & & 1668 & \\
\hline Porcentaje horizontal & 69,7 & & 28,9 & & 1,4 & & 100 & \\
\hline Porcentaje de la PEA total (del cuadro 3) & 19,9 & & 20,7 & & 7,8 & & 19,7 & \\
\hline \multicolumn{9}{|l|}{ Años de escolaridad } \\
\hline Promedio & 8 & & 9,7 & & 11,3 & & 8,6 & \\
\hline Mediana & 9 & & 11 & & 11 & & 10 & \\
\hline
\end{tabular}

Fuente: elaboración propia sobre la base de Encuesta Nacional de Hogares 2003.

\footnotetext{
Población de obreros de 25 años o más (ampliación de la muestra).

Salario medio mensual, en soles, Lima, octubre de 2003.

Número reducido de observaciones, un $10 \%$ o menos del grupo social.

PEA Población económicamente activa.

- No hay población en esta categoría.
} 


\begin{tabular}{|c|c|c|c|c|c|c|c|c|}
\hline \multirow{3}{*}{ Nivel de educación } & \multicolumn{6}{|c|}{ Grupo social } & \multirow{2}{*}{\multicolumn{2}{|c|}{ Total }} \\
\hline & \multicolumn{2}{|c|}{$\mathrm{Z}$} & \multicolumn{2}{|c|}{$\mathrm{Y}$} & \multicolumn{2}{|c|}{$\mathrm{A}$} & & \\
\hline & $\mathrm{N}^{\mathrm{a}}(\%)$ & $\mathrm{IM}^{\mathrm{b}}$ & $\mathrm{N}(\%)$ & $\mathrm{IM}^{\mathrm{b}}$ & $\mathrm{N}(\%)$ & $\mathrm{IM}^{\mathrm{b}}$ & $\mathrm{N}(\%)$ & $\mathrm{IM}^{\mathrm{b}}$ \\
\hline Ninguno & $0,1^{\mathrm{c}}$ & 357 & $0,5^{\mathrm{c}}$ & 431 & - & - & 0,3 & 415 \\
\hline Básica & $4,6^{\mathrm{c}}$ & 703 & $1,8^{\mathrm{c}}$ & 641 & - & - & 2,8 & 685 \\
\hline Secundaria & 29,2 & 871 & 28,1 & 707 & 14,2 & 763 & 27,2 & 788 \\
\hline Técnica & 32,9 & 1045 & 26,2 & 1009 & 17,7 & 1102 & 28,4 & 1033 \\
\hline Universitaria & 33,2 & 1780 & 43,4 & 2203 & 68,1 & 3182 & 41,3 & 2212 \\
\hline Total & 100 & 1222 & 100 & 1433 & 100 & 2469 & 100 & 1442 \\
\hline Total N & 807 & & 833 & & 181 & & 1821 & \\
\hline Porcentaje horizontal & 44,3 & & 45,7 & & 10 & & 100 & \\
\hline Porcentaje de la PEA total (del cuadro 3 ) & 13,8 & & 35,7 & & 61,8 & & 21,5 & \\
\hline \multicolumn{9}{|l|}{ Años de escolaridad } \\
\hline Promedio & 13,5 & & 13,7 & & 14,9 & & 13,7 & \\
\hline Mediana & 14 & & 14 & & 16 & & 14 & \\
\hline
\end{tabular}

Fuente: elaboración propia sobre la base de Encuesta Nacional de Hogares 2003.

Población de empleados de 25 años o más (ampliación de la muestra).

Sueldo medio mensual en soles, Lima, octubre de 2003.

Número reducido de observaciones, un $10 \%$ o menos del grupo social.

PEA Población económicamente activa.

- $\quad$ No hay población en esta categoría.

\section{H4. Hipótesis de la brecha que necesariamente existe entre los ingresos por concepto de salarios y del trabajo por cuenta propia}

Cabe preguntarse cómo aseguran las empresas la disciplina laboral en una sociedad sigma. Como se dijo en la sección II, la teoría sigma parte de la base de que las relaciones laborales de una empresa son conflictivas, de manera que los empleadores deben encontrar formas de lograr que los trabajadores se esfuercen en su trabajo. Por lo tanto, procurarán pagar a los trabajadores contratados salarios de mercado superiores al costo de oportunidad, de manera que aquellos que sean sorprendidos faltando a sus deberes serán despedidos y tendrán que enfrentar un costo económico, representado por su prima salarial. Para los trabajadores contratados por las empresas, el costo de oportunidad será igual a los ingresos que puedan obtener trabajando por cuenta propia. Esta diferencia es el mecanismo de disciplina laboral que las empresas capitalistas utilizan para asegurar el nivel más alto de productividad de la mano de obra en las sociedades sobrepobladas. En consecuencia, el modelo sigma predice que existe una brecha entre el precio de los servicios laborales que se pagan en el mercado de trabajo (sueldos o salarios) y los ingresos que se obtienen del trabajo por cuenta propia, para un nivel determinado de capital humano.

Supóngase que la situación de equilibrio implique que hay una diferencia del $30 \%$ entre los ingresos por concepto de salarios y del trabajo por cuenta propia. Como el salario medio del grupo social $Y$ es superior al del grupo social $\mathrm{Z}$ (que se muestra anteriormente en H3), los ingresos obtenidos por quienes trabajan por cuenta propia también deben tener el mismo orden. La brecha de eficiencia se aplica por separado a cada grupo social. Esta hipótesis difiere del modelo estándar de Lewis (Lewis, 1954), que pasa por alto el factor identidad étnica, de modo que la brecha de eficiencia es uniforme para todos los trabajadores.

En el cuadro 5 se presentan datos sobre el ingreso medio de los asalariados, de los que ganan un sueldo y de los que trabajan por cuenta propia, separados por grupos sociales. En el grupo social Z, el ingreso global de quienes trabajan por cuenta propia es inferior a los sueldos y salarios. Esta relación también se aplica a los niveles de educación pertinentes. En el cuadro 5 
se advierte que en el grupo social Y las relaciones son similares, pero en el grupo social A ellas parecen menos claras que en los demás grupos. En el nivel de educación pertinente, que son los estudios universitarios, la relación es sin embargo bastante clara. Todas las diferencias observadas son estadísticamente significativas como se observa en el Apéndice, de manera que los datos empíricos no refutan el modelo.

En el gráfico 3 se resumen los resultados. En el grupo Z, el salario medio es superior al ingreso medio del trabajo por cuenta propia en los niveles de educación básica y secundaria, que son las categorías pertinentes para los efectos de la comparación. En el grupo Y, el salario medio es superior a los ingresos medios del trabajo por cuenta propia en los tres niveles de educación: secundario, técnico y universitario.

Para resumir, al parecer en el Perú los mercados de trabajo operan en la forma prevista por el modelo sigma, de modo que los que trabajan por cuenta propia generalmente están subempleados, por lo tanto forman parte de la oferta excedente de mano de obra y preferirían emplearse en el mercado de trabajo al nivel de sueldos y salarios corrientes.

En el cuadro 6 se aportan datos sobre la oferta excedente de trabajo (desempleo y subempleo) por grupo social. En términos de las diferencias entre grupos sociales, los resultados indican que las tasas de mano de
GRÁFICO 3

\section{Perú: relación empírica entre sueldos o salarios y trabajo por cuenta propia, según grupos étnicos}

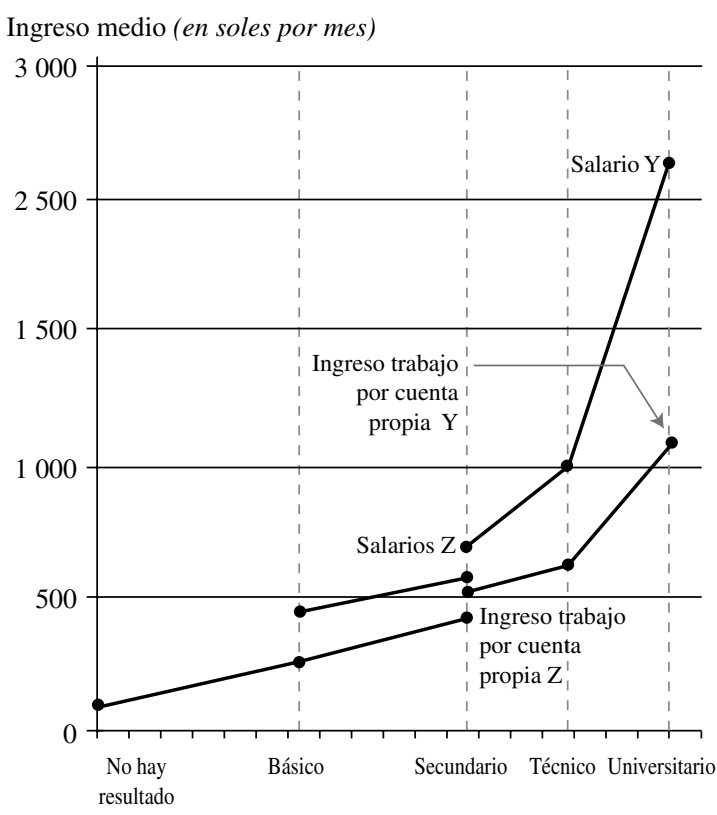

Nivel de educación

Fuente: elaboración propia sobre la base de los datos consignados en el cuadro 4.

CUADRO 5

Perú: promedio salarios, promedio sueldos e ingreso medio del trabajo por cuenta propia, por grupo social, 2003

(En soles por mes)

\begin{tabular}{|c|c|c|c|c|c|c|}
\hline \multirow{2}{*}{ Grupo social } & \multicolumn{5}{|c|}{ Nivel de educación } & \multirow{2}{*}{ Total } \\
\hline & ninguna & básica & secundaria & técnica & universitaria & \\
\hline & \multicolumn{5}{|c|}{ Grupo Z } & \\
\hline Sueldo & $357^{\mathrm{a}}$ & $703^{\mathrm{a}}$ & $871^{\mathrm{a}}$ & $1045^{\mathrm{a}}$ & $1780^{\mathrm{a}}$ & 1222 \\
\hline Salario & $306^{\mathrm{a}}$ & 483 & 618 & $695^{\mathrm{a}}$ & $763^{\mathrm{a}}$ & 557 \\
\hline \multirow[t]{2}{*}{ Ingreso trabajo por cuenta propia } & 136 & 260 & 451 & $568^{\mathrm{a}}$ & $818^{\mathrm{a}}$ & 327 \\
\hline & \multicolumn{5}{|c|}{ Grupo Y } & \\
\hline Sueldo & $431^{\mathrm{a}}$ & $641^{\mathrm{a}}$ & 707 & 1009 & 2203 & 1433 \\
\hline Salario & $349^{\mathrm{a}}$ & $518^{\mathrm{a}}$ & 651 & $712^{\mathrm{a}}$ & $796^{\mathrm{a}}$ & 630 \\
\hline \multirow[t]{2}{*}{ Ingreso trabajo por cuenta propia } & $221^{\mathrm{a}}$ & $384^{\mathrm{a}}$ & 550 & 696 & 1180 & 621 \\
\hline & \multicolumn{5}{|c|}{ Grupo A } & \\
\hline Sueldo & - & - & 763 & 1102 & 3182 & 2469 \\
\hline Salario & - & - & $568^{\mathrm{a}}$ & $743^{\mathrm{a}}$ & - & 608 \\
\hline Ingreso trabajo por cuenta propia & - & $137^{\mathrm{a}}$ & 1032 & $508^{\mathrm{a}}$ & 1135 & 1013 \\
\hline
\end{tabular}

Fuente: elaboración propia sobre la base de Encuesta Nacional de Hogares 2003.

a Número reducido de observaciones, un $10 \%$ o menos del grupo social.

- No hay personas en esta categoría. 
CUADRO 6

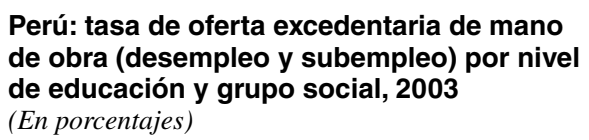

\begin{tabular}{|c|c|c|c|c|}
\hline \multirow{2}{*}{ Nivel de educación } & \multicolumn{3}{|c|}{ Grupo social } & \multirow{2}{*}{ Total } \\
\hline & Z & $\mathrm{Y}$ & A & \\
\hline Ninguno & $\begin{array}{c}88,1 \\
(11,7)^{\mathrm{b}}\end{array}$ & $\begin{array}{r}73,7^{\mathrm{a}} \\
(12,3)\end{array}$ & - & $\begin{array}{c}87,1 \\
(11,7)\end{array}$ \\
\hline Básica & $\begin{array}{l}79,7 \\
(6,5)\end{array}$ & $\begin{array}{l}61,8 \\
(7,2)\end{array}$ & $\begin{array}{r}100,0^{\mathrm{a}} \\
(0,0)\end{array}$ & $\begin{array}{l}77,7 \\
(6,6)\end{array}$ \\
\hline Secundaria & $\begin{array}{l}57,6 \\
(5,4)\end{array}$ & $\begin{array}{l}49,8 \\
(8,2)\end{array}$ & $\begin{array}{c}39,3 \\
(11,4)\end{array}$ & $\begin{array}{l}54,4 \\
(6,5)\end{array}$ \\
\hline Técnica & $\begin{array}{l}35,0^{\mathrm{a}} \\
(5,6)\end{array}$ & $\begin{array}{l}31,1 \\
(7,3)\end{array}$ & $\begin{array}{c}25,7 \\
(12,4)\end{array}$ & $\begin{array}{l}32,9 \\
(6,6)\end{array}$ \\
\hline Universitaria & $\begin{array}{l}29,3^{\mathrm{a}} \\
(7,0)\end{array}$ & $\begin{array}{l}32,7 \\
(8,7)\end{array}$ & $\begin{array}{l}28,8 \\
(2,7)\end{array}$ & $\begin{array}{l}31,0 \\
(7,2)\end{array}$ \\
\hline Total & $\begin{array}{l}65,6 \\
(6,6)\end{array}$ & $\begin{array}{l}44,5 \\
(8,1)\end{array}$ & $\begin{array}{l}31,5 \\
(6,4)\end{array}$ & $\begin{array}{l}58,4 \\
(7,0)\end{array}$ \\
\hline
\end{tabular}

Fuente: elaboración propia sobre la base de Encuesta Nacional de Hogares 2003.

a Número reducido de observaciones, un $10 \%$ o menos del grupo social.

b Tasa de desempleo entre paréntesis; por lo tanto, el subempleo es igual a la diferencia entre la tasa total de oferta excedentaria de mano de obra (indicada en el cuadro) y la tasa de desempleo (entre paréntesis).

- No hay personas en esta categoría.

obra excedente son muy elevadas: $66 \%$ en el grupo social $\mathrm{Z}, 45 \%$ en el grupo social Y y $32 \%$ en el grupo social A. En el Apéndice se aprecia que las diferencias observadas en la oferta excedentaria por nivel de educación y grupo social, en las categorías pertinentes, son estadísticamente significativas. Como las tasas de desempleo no difieren mayormente, esta diferencia indica más que nada las tasas de subempleo.

\section{H5. Hipótesis de alta movilidad en materia de edu- cación, pero baja movilidad de ingresos}

El modelo dinámico sigma predice que el promedio de años de educación tiende a nivelar a los grupos sociales en el tiempo, pero que no sucede lo mismo con los ingresos medios. Esta predicción puede probarse estadísticamente utilizando tramos de edad como indicadores de las diferencias intergeneracionales.

En los cuadros 7A y 7B se muestra la razón entre educación e ingresos por grupos intergeneracionales de trabajadores y grupos sociales, respectivamente. En el cuadro 7A resalta un importante incremento en los años de escolaridad entre generaciones en el grupo social Z: los "hijos" (en el sentido social y no biológico) tienen el doble de años de escolaridad que sus "padres". El aumento generado en otros grupos sociales es menor. En el grupo social A la educación parece tener un tope en torno de los 14 años de escolaridad. Como consecuencia de ello, la brecha en materia de educación entre el grupo $\mathrm{Z}$ y los otros grupos se ha estrechado notoriamente.

Los cambios estimados de las brechas de ingreso a través de las generaciones se presentan en el cuadro 7B. $\mathrm{El}$ ingreso medio del grupo social $\mathrm{Z}$ en relación con el grupo social A no ha variado mucho: el ingreso relativo de los "padres" es del 30\%, mientras que el de los "hijos" asciende al $40 \%$, lo que es poco si se compara con los cambios en materia de logros educacionales mostrados en el cuadro 7A. La situación del grupo social Y respecto del grupo social A sigue un patrón similar. El ingreso relativo de "padres" e "hijos" se mantiene casi constante en torno del $40 \%$ y el $50 \%$, salvo en el caso de los mayores, donde la cifra es del $100 \%$. Este último resultado es ciertamente paradójico, tal vez debido al tamaño reducido de este grupo.

Con respecto a las tendencias de convergencia entre grupos sociales pertenecientes a generaciones diferentes se concluye que - no obstante que las disparidades en años de escolaridad propenden a disminuir- no se da una tendencia análoga a reducir la desigualdad de los ingresos. Este resultado empírico coincide con la hipótesis.

Por lo que respecta a la desigualdad de ingresos en el Perú, el coeficiente de Gini estimado a partir de los datos de la ENAHO 2003 es 0,59, lo que se aproxima a otras estimaciones realizadas en el pasado $(0,60)$ sobre la base de las cuentas nacionales. Esta cifra debe subestimar el verdadero coeficiente de Gini, porque en la muestra no se incluyen los ingresos de la elite económica del Perú. Es un hecho conocido que en las encuestas de hogares no se incluye a las elites económicas o se las subrepresenta. La verdad es que el grupo social empírico A se compone fundamentalmente de la clase media (ejecutivos, administradores y profesionales de alto nivel) y no corresponde a la clase capitalista del modelo. Teóricamente, en la estructura social dual de capitalistas y trabajadores, la clase media suele incluirse en la categoría capitalista (Wright, 1997), criterio que se aplicó en el presente trabajo.

En el Perú sigue existiendo un grado relativamente alto de desigualdad y el país continúa siendo uno 
Perú: años de escolaridad por tramo de edad y grupo social, 2003

\begin{tabular}{|c|c|c|c|c|c|c|c|c|c|c|}
\hline \multirow{3}{*}{ Grupo social } & \multicolumn{8}{|c|}{ Tramo de edad (años) } & \multirow{2}{*}{\multicolumn{2}{|c|}{ Total }} \\
\hline & \multicolumn{2}{|c|}{$25-34$} & \multicolumn{2}{|c|}{$35-44$} & \multicolumn{2}{|c|}{$45-54$} & \multicolumn{2}{|c|}{$55-65$} & & \\
\hline & $\mathrm{N}^{\mathrm{a}}$ & $\mathrm{E}^{\mathrm{b}}$ & $\mathrm{N}$ & $\mathrm{E}$ & $\mathrm{N}$ & $\mathrm{E}$ & $\mathrm{N}$ & $\mathrm{E}$ & $\mathrm{N}$ & $\mathrm{E}$ \\
\hline $\mathrm{Z}$ & 1551 & 11 & 1636 & 8 & 1260 & 7 & 850 & 5 & 5297 & 8 \\
\hline Y & 912 & 12 & 670 & 12 & 449 & 11 & 218 & 9 & 2249 & 12 \\
\hline A & 146 & 14 & 111 & 14 & 29 & 14 & 6 & 12 & 291 & 14 \\
\hline Total & 2609 & 12 & 2417 & 10 & 1738 & 8 & 1074 & 6 & 7837 & 9 \\
\hline Razón Z/A & 0,8 & & 0, & & 0,5 & & 0,4 & & 0,6 & \\
\hline Razón Y/A & 0, & & 0,8 & & 0,8 & & 0,7 & & 1,2 & \\
\hline
\end{tabular}

Fuente: elaboración propia sobre la base de Encuesta Nacional de Hogares 2003.

a Población económicamente activa empleada (miles de personas).

b Promedio años de escolaridad.

CUADRO 7B

Perú: ingreso medio por tramo de edad y grupo social, 2003

\begin{tabular}{|c|c|c|c|c|c|c|c|c|c|c|}
\hline \multirow{3}{*}{ Grupo social } & \multicolumn{8}{|c|}{ Tramo de edad (años) } & \multirow{2}{*}{\multicolumn{2}{|c|}{ Total }} \\
\hline & \multicolumn{2}{|c|}{$25-34$} & \multicolumn{2}{|c|}{$35-44$} & \multicolumn{2}{|c|}{$45-54$} & \multicolumn{2}{|c|}{$55-65$} & & \\
\hline & $\mathrm{N}^{\mathrm{a}}$ & $\mathrm{y}^{\mathrm{b}}$ & $\mathrm{N}$ & $\mathrm{y}$ & $\mathrm{N}$ & $\mathrm{y}$ & $\mathrm{N}$ & $\mathrm{y}$ & $\mathrm{N}$ & $\mathrm{y}$ \\
\hline $\mathrm{Z}$ & 1551 & 530 & 1636 & 596 & 1260 & 623 & 850 & 473 & 5297 & 563 \\
\hline $\mathrm{Y}$ & 912 & 771 & 670 & 987 & 449 & 1195 & 218 & 1626 & 2249 & 1003 \\
\hline A & 146 & 1456 & 111 & 2455 & 29 & 2982 & 6 & 1648 & 291 & 1991 \\
\hline Total & 2609 & 666 & 2417 & 790 & 1738 & 810 & 1074 & 713 & 7837 & 743 \\
\hline Razón Z/A & 0,4 & & 0,2 & & 0,2 & & 0,3 & & 0,3 & \\
\hline Razón Y/A & 0,5 & & 0 & & 0,4 & & 1,0 & & 0,5 & \\
\hline
\end{tabular}

Fuente: elaboración propia sobre la base de Encuesta Nacional de Hogares 2003.

a Población económicamente activa empleada (miles de personas).

b Ingreso medio (soles por mes).

de los más desiguales del mundo. Como predice el modelo sigma, la educación no parece ser un sistema nivelador de los ingresos. Aun cuando las personas tengan el mismo número de años de escolaridad, su ingreso medio siempre dependerá del grupo social a que pertenezcan. 


\section{VII}

\section{Conclusiones}

El modelo sigma construido en este trabajo predice un conjunto de hipótesis empíricamente refutables respecto de las relaciones entre educación e ingresos. Al compararlas con los datos correspondientes al Perú, las pruebas estadísticas evidencian que las hipótesis no se rechazan. El modelo sigma también predice la paradoja que se observa en los países en desarrollo, donde un incremento significativo del número de años de escolaridad no ha ido acompañado de una menor desigualdad en materia de ingresos.
La conclusión de que la educación no es un sistema nivelador de los ingresos se explica por dos factores: la desigualdad inicial de la distribución de activos económicos y políticos entre grupos sociales (alto grado de desigualdad) y la dotación de factores de la sociedad (sobrepoblación). La desigualdad inicial y la sobrepoblación son los factores que en definitiva explican la paradoja. En otras palabras, son las variables exógenas del modelo sigma, de tal manera que mientras estas variables exógenas no varíen el sistema educativo seguirá siendo no nivelador.

APÉNDICE

Resumen de las pruebas estadísticas

Hipótesis 1: Diferencia en el promedio de años de escolaridad

\begin{tabular}{|c|c|c|c|c|c|c|c|}
\hline \multirow{2}{*}{ Grupos sociales } & \multicolumn{2}{|c|}{ Prueba igualdad de varianza } & \multicolumn{5}{|c|}{ Diferencia de la prueba de valores medios } \\
\hline & Estadísticas T & Valor $p$ & Diferencia & \multicolumn{2}{|c|}{ Intervalo de confianza } & \multirow{2}{*}{$\frac{F}{2024,4}$} & \multirow{2}{*}{$\frac{\text { Valor } p}{0}$} \\
\hline $\mathrm{Z}-\mathrm{Y}$ & 354,56 & 0,00 & $-3,96$ & {$[-4,10$} & $-3,81]$ & & \\
\hline $\mathrm{Z}-\mathrm{A}$ & 150,21 & 0,00 & $-6,87$ & {$[-7,20$} & $-6,55]$ & 1620,48 & 0 \\
\hline \multirow[t]{2}{*}{$\mathrm{Y}-\mathrm{A}$} & 53,21 & 0,00 & $-2,92$ & {$[-3,26$} & $-2,58]$ & 258,3 & 0 \\
\hline & \multicolumn{7}{|c|}{ Prueba U de Mann-Whitney } \\
\hline \multirow{2}{*}{ Grupos sociales } & \multicolumn{3}{|c|}{ Suma de categorías } & \multirow{2}{*}{\multicolumn{3}{|c|}{ Estadísticas Z }} & \multirow{2}{*}{ Valor $p$} \\
\hline & \multicolumn{2}{|l|}{$\mathrm{Z}$} & Y & & & & \\
\hline$Z-Y$ & \multicolumn{2}{|c|}{490300000} & 218000000 & \multicolumn{3}{|c|}{$-61,49$} & 0,00 \\
\hline $\mathrm{Z}-\mathrm{A}$ & \multirow{2}{*}{\multicolumn{2}{|c|}{$\begin{array}{r}420400000 \\
38984586\end{array}$}} & \multirow{2}{*}{\multicolumn{2}{|c|}{$\begin{array}{r}10058291 \\
2479585\end{array}$}} & \multicolumn{2}{|c|}{$-24,62$} & 0,00 \\
\hline$Y-A$ & & & & & \multicolumn{2}{|c|}{$-12,57$} & 0,00 \\
\hline
\end{tabular}

Fuente: elaboración del autor.

Las pruebas estadísticas se refieren a las diferencias en el promedio de años de escolaridad entre los grupos sociales. En la primera prueba se utilizan estadísticas F, y en la segunda prueba, U (no paramétrica) de Mann-Whitney. Los resultados indican que las diferencias observadas de la media son estadísticamente significativas. 
Hipótesis 2: promedio diferencias de ingreso

\begin{tabular}{|c|c|c|c|c|c|c|c|c|}
\hline \multirow{2}{*}{$\begin{array}{l}\text { Nivel de } \\
\text { educación }\end{array}$} & \multirow{2}{*}{$\begin{array}{l}\text { Grupos } \\
\text { sociales }\end{array}$} & \multicolumn{2}{|c|}{ Prueba igualdad de varianza } & \multicolumn{5}{|c|}{ Diferencia de la prueba de valores medios } \\
\hline & & Estadísticas T & Valor $p$ & Diferencia & Intervalo d & onfianza & Estadísticas F & Valor $p$ \\
\hline Básica & $\mathrm{Z}-\mathrm{Y}$ & 4,24 & 0,04 & $-126,41$ & {$[-164,86$} & $-87,97]$ & 33,04 & 0,00 \\
\hline \multirow{3}{*}{ Secundaria } & $Z-Y$ & 0,63 & 0,43 & $-102,02$ & {$[-162,35$} & $-41,68]$ & 6,35 & 0,01 \\
\hline & $\mathrm{Z}-\mathrm{A}$ & 0,15 & 0,70 & $-217,56$ & {$[-513,60$} & $78,47]$ & 2,11 & 0,15 \\
\hline & $\mathrm{Y}-\mathrm{A}$ & 0,07 & 0,80 & $-115,54$ & {$[-416,41$} & $185,32]$ & 0,35 & 0,55 \\
\hline Técnica & $\mathrm{Y}-\mathrm{A}$ & 0,96 & 0,33 & $-41,85$ & {$[-307,86$} & 224,17] & 0,15 & 0,69 \\
\hline Universitaria & $\mathrm{Y}-\mathrm{A}$ & 21,48 & 0,00 & $-938,12$ & {$[-1677,40$} & $-198,83]$ & 3,30 & 0,07 \\
\hline
\end{tabular}

\begin{tabular}{|c|c|c|c|c|c|}
\hline \multirow{3}{*}{ Nivel de educación } & \multirow{3}{*}{ Grupos sociales } & \multicolumn{4}{|c|}{ Prueba U de Mann-Whitney } \\
\hline & & \multicolumn{2}{|c|}{ Suma de categorías } & \multirow{2}{*}{ Estadísticas Z } & \multirow{2}{*}{ Valor $p$} \\
\hline & & $\mathrm{Z}$ & $\mathrm{Y}$ & & \\
\hline Básica & $Z-Y$ & 34224589 & 4808441 & $-9,83$ & 0,00 \\
\hline Secundaria & $\begin{array}{l}Z-Y \\
Z-A \\
Y-A\end{array}$ & $\begin{array}{r}23098507 \\
17017958 \\
3033103\end{array}$ & $\begin{array}{r}10714470 \\
289828 \\
113184\end{array}$ & $\begin{array}{l}-7,61 \\
-2,76 \\
-1,20\end{array}$ & $\begin{array}{l}0,00 \\
0,01 \\
0,23\end{array}$ \\
\hline Técnica & $\mathrm{Y}-\mathrm{A}$ & 553451 & 31370 & $-0,72$ & 0,47 \\
\hline Universitaria & $\mathrm{Y}-\mathrm{A}$ & 785177 & 103934 & $-0,66$ & 0,00 \\
\hline
\end{tabular}

Fuente: elaboración del autor.

La prueba se refiere ahora a las diferencias de los valores medios de los ingresos entre grupos sociales. Las pruebas paramétricas y no paramétricas indican que las diferencias observadas son estadísticamente significativas en los niveles de educación básica y universitaria. En los demás niveles de educación la prueba paramétrica no pasa la prueba de la igualdad de varianza y, en consecuencia, corresponde aplicar las pruebas paramétricas. Estas indican que las diferencias no son estadísticamente significativas a nivel de la educación técnica, mientras que a nivel de la enseñanza secundaria las diferencias son significativas en todos los grupos, salvo Y-A. En cuatro de seis casos, los hechos no refutan la hipótesis.

Hipótesis 3: Diferencias en el promedio de los sueldos y de los salarios

Empleados: sueldos

\begin{tabular}{|c|c|c|c|c|c|c|c|c|}
\hline \multirow{2}{*}{ Nivel de educación } & \multirow{2}{*}{$\begin{array}{l}\text { Grupos } \\
\text { sociales }\end{array}$} & \multicolumn{2}{|c|}{$\begin{array}{l}\text { Prueba de igualdad } \\
\text { de la varianza }\end{array}$} & \multicolumn{5}{|c|}{$\begin{array}{l}\text { Diferencia de la prueba } \\
\text { de valores medios }\end{array}$} \\
\hline & & Estadísticas T & Valor $p$ & Diferencia & Intervalo de & onfianza & Estadísticas F & Valor $p$ \\
\hline Secundaria & $\begin{array}{l}Z-Y \\
Z-A \\
Y-A\end{array}$ & $\begin{array}{l}8,73 \\
1,71 \\
0,39\end{array}$ & $\begin{array}{l}0,00 \\
0,19 \\
0,53\end{array}$ & $\begin{array}{l}164,01 \\
107,76 \\
-56,25\end{array}$ & $\begin{array}{r}{[93,99} \\
{[-143,00} \\
{[-307,71}\end{array}$ & $\begin{array}{l}234,03] \\
358,52] \\
195,21]\end{array}$ & $\begin{array}{r}21,08 \\
0,71 \\
0,19\end{array}$ & $\begin{array}{l}0,00 \\
0,40 \\
0,66\end{array}$ \\
\hline Técnica & $\begin{array}{l}Z-Y \\
Z-A \\
Y-A\end{array}$ & $\begin{array}{l}4,75 \\
0,70 \\
0,00\end{array}$ & $\begin{array}{l}0,03 \\
0,40 \\
0,97\end{array}$ & $\begin{array}{r}35,85 \\
-56,94 \\
-92,79\end{array}$ & $\begin{array}{r}{[-67,13} \\
{[-378,31} \\
{[-421,73}\end{array}$ & $\begin{array}{l}138,83] \\
264,43] \\
236,15]\end{array}$ & $\begin{array}{l}0,47 \\
0,12 \\
0,31\end{array}$ & $\begin{array}{l}0,50 \\
0,73 \\
0,58\end{array}$ \\
\hline Universitaria & $\begin{array}{l}Z-Y \\
Z-A \\
Y-A\end{array}$ & $\begin{array}{l}21,64 \\
60,13 \\
16,44\end{array}$ & $\begin{array}{l}0,00 \\
0,00 \\
0,00\end{array}$ & $\begin{array}{r}-422,79 \\
-1402,16 \\
-979,37\end{array}$ & 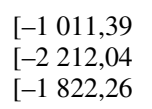 & $\begin{array}{r}165,82] \\
-592,28] \\
-136,48]\end{array}$ & $\begin{array}{r}1,98 \\
11,52 \\
5,19\end{array}$ & $\begin{array}{l}0,16 \\
0,00 \\
0,02\end{array}$ \\
\hline
\end{tabular}


(continuación tabla anterior)

\begin{tabular}{|c|c|c|c|c|c|}
\hline \multirow{3}{*}{ Nivel de educación } & \multirow{3}{*}{ Grupos sociales } & \multicolumn{4}{|c|}{ Prueba U de Mann-Whitney } \\
\hline & & \multicolumn{2}{|c|}{ Suma de categorías } & \multirow{2}{*}{ Estadísticas Z } & \multirow{2}{*}{ Valor $p$} \\
\hline & & Z & $\mathrm{Y}$ & & \\
\hline \multirow[t]{3}{*}{ Secundaria } & Z-Y & 476879 & 271598 & 4,27 & 0,00 \\
\hline & Z-A & 284144 & 8852 & 1,63 & 0,10 \\
\hline & $\mathrm{Y}-\mathrm{A}$ & 125362 & 6994 & 0,28 & 0,78 \\
\hline \multirow[t]{4}{*}{ Técnica } & Z-Y & 667938 & 391302 & 3,39 & 0,00 \\
\hline & Z-A & 405470 & 13601 & 1,30 & 0,19 \\
\hline & Y-A & 175132 & 10004 & 0,35 & 0,73 \\
\hline & $\mathrm{Z}-\mathrm{Y}$ & 646406 & 660130 & $-1,85$ & 0,06 \\
\hline \multirow[t]{2}{*}{ Universitaria } & Z-A & 363848 & 56139 & $-5,15$ & 0,00 \\
\hline & Y-A & 344454 & 52041 & $-4,10$ & 0,00 \\
\hline
\end{tabular}

Fuente: elaboración del autor.

Dado el valor relativo de los grupos sociales (que figuran en la primera hipótesis sobre exclusión), lo que corresponde comparar es entre los grupos Z-Y-A en los niveles de educación secundaria, técnica y universitaria. El contraste con el nivel universitario puede hacerse mediante pruebas paramétricas y no paramétricas. Debido a que no se pasa la prueba de igualdad de la varianza, los otros dos niveles pueden someterse exclusivamente a la prueba no paramétrica. Los resultados indican que las diferencias observadas en el nivel de enseñanza universitaria son estadísticamente significativas de manera sostenida; en general, los valores observados son estadísticamente significativos en cinco de los nueve casos.

\section{Obreros: salarios}

\begin{tabular}{|c|c|c|c|c|c|c|c|c|}
\hline \multirow{2}{*}{ Nivel de educación } & \multirow{2}{*}{$\begin{array}{l}\text { Grupos } \\
\text { sociales }\end{array}$} & \multicolumn{2}{|c|}{$\begin{array}{l}\text { Prueba de igualdad } \\
\text { de la varianza }\end{array}$} & \multicolumn{5}{|c|}{$\begin{array}{l}\text { Diferencia de la prueba } \\
\text { de valores medios }\end{array}$} \\
\hline & & Estadísticas T & Valor $p$ & Diferencia & Intervalo & onfianza & Estadísticas F & Valor $p$ \\
\hline Básica & $Z-Y$ & 0,38 & 0,54 & $-35,08$ & {$[-115,23$} & $45,07]$ & 0,74 & 0,39 \\
\hline Secundaria & $\mathrm{Z}-\mathrm{Y}$ & 4,01 & 0,05 & $-34,34$ & {$[-91,07$} & $22,40]$ & 1,41 & 0,24 \\
\hline
\end{tabular}

\begin{tabular}{lccccc}
\hline & & \multicolumn{3}{c}{ Prueba U de Mann-Whitney } \\
\cline { 3 - 5 } Nivel de educación & Grupos sociales & \multicolumn{2}{c}{ Suma de categorías } & Estadísticas Z & Valor $p$ \\
\cline { 3 - 5 } & & $\mathrm{Z}$ & $\mathrm{Y}$ & $-0,93$ & 0,35 \\
Básica & $\mathrm{Z}-\mathrm{Y}$ & 1139292 & 212899 & $-2,71$ & 0,01 \\
Secundaria & $\mathrm{Z}-\mathrm{Y}$ & 1653321 & 774385 & & \\
\hline
\end{tabular}

Fuente: elaboración del autor.

Corresponde comparar entre los grupos Z-Y en los niveles básico y secundario. No se puede utilizar la prueba paramétrica debido a que falla la prueba de igualdad de la varianza. La prueba no paramétrica denota que las diferencias observadas son estadísticamente significativas en el nivel de enseñanza secundaria (que compone la mayor parte del grupo), pero no a nivel de la enseñanza básica. 
Hipótesis 4: Diferencia de los valores medios entre los ingresos por concepto de salarios y los ingresos del trabajo por cuenta propia

Grupo social Z

\begin{tabular}{|c|c|c|c|c|c|c|c|}
\hline \multirow{2}{*}{ Nivel de educación } & \multicolumn{2}{|c|}{$\begin{array}{l}\text { Prueba de igualdad de la } \\
\text { varianza }\end{array}$} & \multicolumn{5}{|c|}{$\begin{array}{l}\text { Diferencia de la prueba } \\
\text { de valores medios }\end{array}$} \\
\hline & Estadísticas T & Valor $p$ & Diferencia & Interval & confianza & Estadísticas F & Valor $p$ \\
\hline Básica & 72,56 & 0,00 & 443,13 & {$[287,90$} & $598,36]$ & 31,31 & 0,00 \\
\hline Secundaria & 24,64 & 0,00 & 420,17 & {$[365,23$} & $475,12]$ & 224,64 & 0,00 \\
\hline Total & 495,77 & 0,00 & 894,33 & {$[757,69$} & $1030,97]$ & 164,58 & 0,00 \\
\hline
\end{tabular}

Prueba U de Mann-Whitney

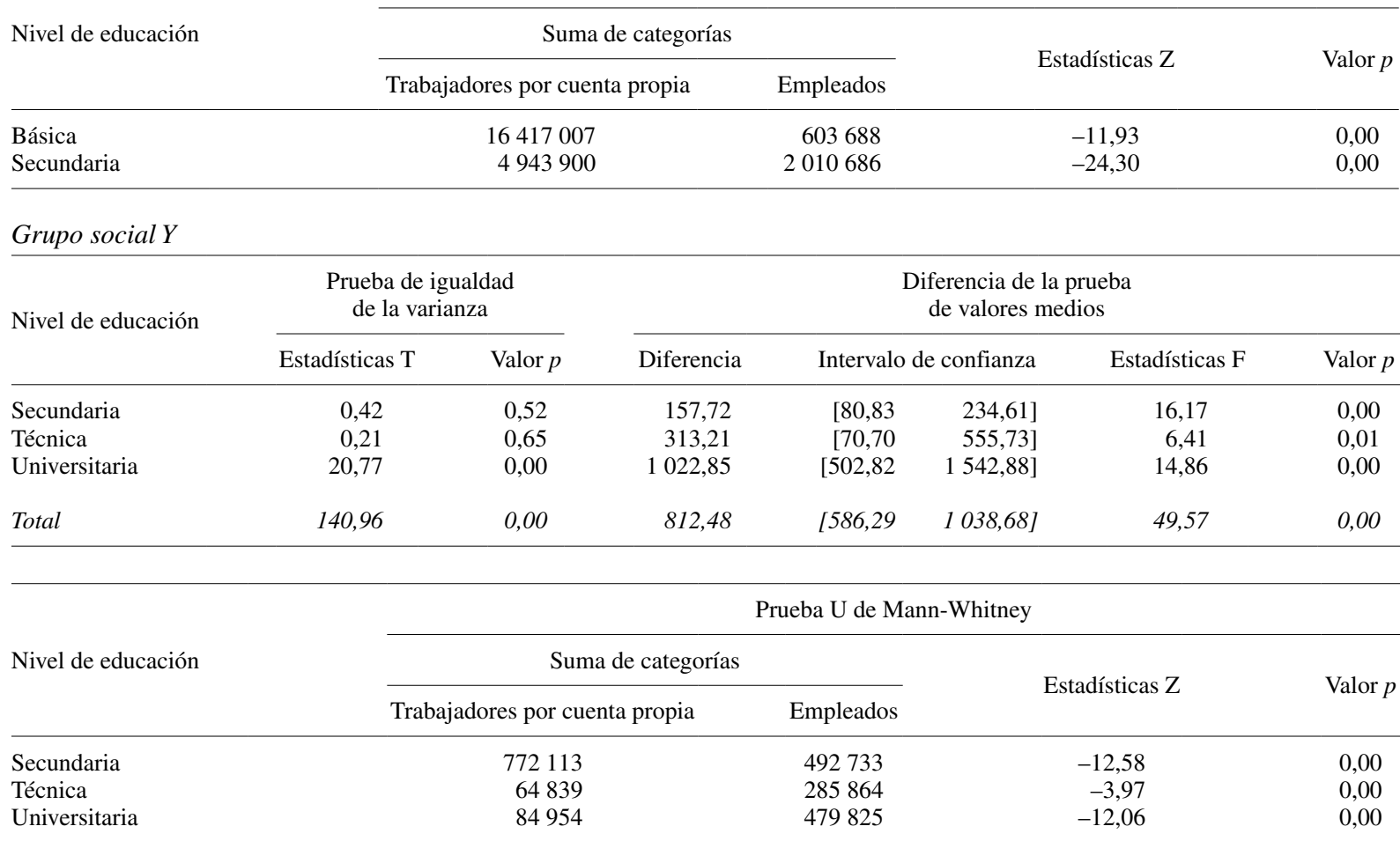

Fuente: elaboración del autor.

En el caso de los trabajadores Z, en las comparaciones aplicables se incluye aquella entre los niveles de educación básica y secundaria. Las pruebas paramétrica y no paramétrica indican que las diferencias observadas son estadísticamente significativas. En el caso de los trabajadores Y, las comparaciones aplicables incluyen los niveles de educación secundaria, técnica y universitaria. La prueba no paramétrica muestra que en todos los casos las diferencias observadas son estadísticamente significativas. La prueba paramétrica solo es aplicable a nivel universitario, en el que también indica que la diferencia es significativa. 
Hipótesis 5: Diferencia de los valores medios en las tasas de exclusión de los mercados de trabajo

\begin{tabular}{lccc}
\hline Nivel de educación & Grupos sociales & Valor chi-cuadrado & Valor $p$ \\
\hline Básica & Z - Y & 6855 & 0,00 \\
Secundaria & Z - Y - A & 4855 & 0,00 \\
Técnica & Z - Y & 1034 & 0,00 \\
Universitaria & Z - Y & 819 & 0,00 \\
\hline
\end{tabular}

Fuente: elaboración del autor.

La prueba del valor chi-cuadrado se utiliza para probar la diferencia de la proporción de exclusión del mercado de trabajo entre grupos sociales. Los resultados indican que las diferencias observadas son estadísticamente significativas en todos los niveles de educación y grupos sociales pertinentes en cada nivel de educación.

Bibliografía

Akerlof, G. y R. Kranton (2000), "Economics and identity", The Quarterly Journal of Economics, vol. 115, № 3, Cambridge, Massachusetts, The MIT Press.

Banco Mundial (2001), World Development Report. Attacking Poverty, Washington, D.C.

Becker, G. y N. Tomes (1979), "An equilibrium theory of the distribution of income and intergenerational mobility", Journal of Political Economy, vol. 87, $\mathrm{N}^{\circ}$ 6, Chicago, University of Chicago Press.

Birdsall, N., A. de la Torre y R. Menezes (2008), Fair Growth: Economic Policies for Latin America's Poor and Middle-Income Majority, Washington, D.C., Brookings Institution Press.

Blom, A. y C. Vélez (2004), "The dynamics of the skill-premium in Brazil: growing demand and insufficient supply?", Inequality and Economic Development in Brazil, Washington, D.C., Banco Mundial.

Bourguignon, F., F. Ferreira y M. Menéndez (2007), "Inequality of opportunity in Brazil", Review of Income and Wealth, vol. 53, $\mathrm{N}^{\circ}$ 4, Oxford, Blackwell Publishing.

Cueto, S., E. Jacoby y E. Pollit (1997), "Rendimiento escolar de niños y niñas en zonas rurales y urbanas del Perú", Revista de psicología, vol. 15, $\mathrm{N}^{\mathrm{o}}$ 1, Lima, Pontificia Universidad Católica del Perú.

Deininger, K. y L. Squire (1996), "A new data set measuring inequality", The World Bank Economic Review, vol. 10, № 3, Washington, D.C., Banco Mundial.

Durlauf, S. (1996), "A theory of persistent income inequality", Journal of Economic Growth, vol. 1, № 1, Nueva York, Springer.

Gardner, H. (1999), Intelligence Reframed: Multiple Intelligences for the $21^{\text {st }}$ Century, Nueva York, Basic Books.
Hall, G. y A. Patrinos (2006), Indigenous Peoples, Poverty, and Human Development in Latin America, Londres, Palgrave.

Hudson, R.A. (1996), Sociolinguistics, Cambridge, Cambridge University Press.

Lewis, A. (1954), "Economic development with unlimited supplies of labor", The Manchester School of Economic and Social Studies, vol. 22, Oxford, Blackwell Publishing.

Li, H., L. Squire y H. Zou (1998), "Explaining international and intertemporal variations in income inequality", The Economic Journal, vol. 108, № 1, Oxford, Blackwell Publishing.

Marshall, T.H. y T. Bottomore (1992), Citizenship and Social Class, Londres, Pluto Press.

Mincer, J. (1974), Schooling, Experience, and Earnings, Cambridge, Massachusetts, National Bureau of Economic Research.

Ñopo, H., J. Saavedra y M. Torero (2004), "Ethnicity and earnings in urban Peru", IZA Discussion Papers, N 980, Bonn, Institute for the Study of Labor (IZA).

Perú, Ministerio de Educación (2005), Evaluación nacional 2004, Lima. Ratey, J. (2002), A User's Guide to the Brain, Nueva York, Vintage Books.

Rivera, I. (1979), "Los determinantes de la calidad de la educación en el Perú. Un Estudio ECIEL", Documento de trabajo, № 44, Lima, Departamento de Economía, Pontificia Universidad Católica del Perú.

Rousseau, J. (1984), A Discourse on Inequality, traducido del francés por Maurice Cranston, Nueva York, Penguin Books. Publicado originalmente en 1755 .

Searle, J. (1995), The Construction of Social Reality, Nueva York, The Free Press.

Wright, E. (1997), Class Counts: Comparative Studies in Class Analysis, Cambridge, Cambridge University Press. 